\title{
openheart Protective lipid-lowering variants in healthy older individuals without coronary heart disease
}

\author{
Paul Lacaze (D) , ${ }^{1}$ Moeen Riaz, ${ }^{1}$ Robert Sebra, ${ }^{2}$ Amanda J Hooper, ${ }^{3,4}$ \\ Jing Pang (D) , ${ }^{3}$ Jane Tiller, ${ }^{1}$ Galina Polekhina, ${ }^{1}$ Andrew Tonkin, ${ }^{1}$ Chris Reid, ${ }^{1,5}$ \\ Sophia Zoungas, ${ }^{1}$ Anne M Murray, ${ }^{6}$ Stephen Nicholls, ${ }^{7}$ Gerald Watts, ${ }^{4,8}$ \\ Eric Schadt, ${ }^{2}$ John J McNeil ${ }^{1}$
}

\begin{abstract}
- Additional supplemental material is published online only. To view, please visit the journal online (http://dx.doi.org/10. 1136/openhrt-2021-001710).
\end{abstract}

To cite: Lacaze P, Riaz M, Sebra $\mathrm{R}$, et al. Protective lipidlowering variants in healthy older individuals without coronary heart disease. Open Heart 2021;8:e001710. doi:10.1136/ openhrt-2021-001710

Received 3 May 2021 Accepted 13 July 2021
Check for updates

(C) Author(s) (or their employer(s)) 2021. Re-use permitted under CC BY-NC. No commercial re-use. See rights and permissions. Published by BMJ.

For numbered affiliations see end of article.

Correspondence to Dr Paul Lacaze; paul.lacaze@ monash.edu

\section{ABSTRACT}

Objective Genetic variants that disrupt the function of the PCSK9 (proprotein convertase subtilisin kexin type 9) and $A P O B$ (apolipoprotein B)genes result in lower serum low-density lipoprotein cholesterol (LDL-C) levels and subsequently confer protection against coronary heart disease (CHD). The objective of this study was to measure the prevalence and selective advantage of such variants among healthy older individuals without a history of CHD. Methods We performed targeted sequencing of the PCSK9 and $A P O B$ genes in 13131 healthy individuals without CHD aged 70 years or older enrolled into the ASPirin in Reducing Events in the Elderly trial. We detected variants in the $P C S K 9$ and $A P O B$ genes with predicted loss-of-function. We associated variant carrier status with serum LDL-C and total cholesterol (TC) levels at the time of study enrolment, adjusting for statin use.

Results We detected 22 different rare PCSK9/APOB candidate variants with putative lipid-lowering effect, carried by 104 participants (carrier rate 1 in 126). Serum LDL-C and TC concentrations for rare PCSK9/APOB variant carriers were consistently lower than non-carriers. Rare variant carrier status was associated with $19.4 \mathrm{mg} /$ $\mathrm{dL}(14.6 \%)$ lower LDL-C, compared with non-carriers $(p \leq 0.001$, adjusted for statin use). Statin prescriptions were less prevalent in rare variant carriers $(16 \%)$ than non-carriers (35\%). The more common PCSK9 R46L variant (rs11591147-T) was associated with $15.5 \mathrm{mg} / \mathrm{dL}$ (11.8\%) lower LDL-C in heterozygotes, and $25.2 \mathrm{mg} / \mathrm{dL}$ $(19.2 \%)$ lower LDL-C in homozygotes (both $p \leq 0.001$ ). Conclusions Lipid-lowering genetic variants are carried by healthy older individuals and contribute to $\mathrm{CHD}$-free survival.

Trial registration number NCT01038583.

\section{INTRODUCTION}

Genetic variants that lower serum low-density lipoprotein cholesterol (LDL-C) levels have been demonstrated to be protective against coronary heart disease (CHD) ${ }^{1-9}$ In particular, protection can be conferred by rare loss-offunction (LoF), protein-truncating variants in canonical lipid-metabolism genes, including the apolipoprotein $\mathrm{B}(A P O B)^{3}$ and the proprotein

\section{Key questions}

What is already known about this subject?

- Loss-of-function genetic variants in the PCSK9 (proprotein convertase subtilisin kexin type 9) and $A P O B$ (apolipoprotein B) genes result in lower serum lowdensity lipoprotein cholesterol concentrations and subsequently confer protection against coronary heart disease (CHD).

What does this study add?

- Our study measured the prevalence and selective advantage of lipid-lowering genetic variants in a unique population of healthy older individuals aged $\geq 70$ years without a history of CHD events.

How might this impact on clinical practice?

- An improved understanding of the role played by protective lipid-lowering variants may help inform future approaches to CHD risk-reduction and lipid management.

convertase subtilisin kexin type $9{\text { (PCSK9 })^{3} 10}^{10}$ genes. These protective lipid-lowering genetic variants tend to be rare in the general population. In addition, LDL-C particle size and other lipoprotein-related genotypes have been associated with CHD-free longevity. ${ }^{11}$

Familial hypobetalipoproteinaemia is caused by heterozygosity for $A P O B$ variants that generally result in LDL-C concentrations that are $>50 \%$ lower than normal, while PCSK9 LoF variants are associated with more modest effects of $15 \%-40 \%$ lower LDL-C. ${ }^{12}$ Discovery and understanding of rare protective variants in these genes, particularly PCSK9, has informed the successful development of several lipid-lowering therapies. ${ }^{13}$ However, most cholesterol-lowering variants, to date, have been identified from casecontrol or population-based studies. ${ }^{1-9} \mathrm{Popu}-$ lations of healthy older individuals without a history of CHD represent an understudied resource for the discovery and understanding of protective lipid-modifying genetic variants. 
With this rationale, we sequenced 13131 healthy older individuals aged $\geq 70$ years without a previous history of diagnosed CHD events, enrolled in the ASPirin in Reducing Events in the Elderly (ASPREE) trial. ${ }^{14}$ Among this healthy older CHDfree population (average age 75 years), we hypothesised that the prevalence of rare cholesterol-lowering variants in canonical lipid metabolism genes (PCSK9/APOB) would be enriched, and that variant carrier status would be associated with lower serum LDL-C and total cholesterol (TC) levelssubsequently contributing to CHD-free survival.

\section{METHODS \\ Study population}

Participants were enrolled in the ASPREE study, a randomised, placebo-controlled trial of daily low-dose aspirin investigating the effect of daily $100 \mathrm{mg}$ aspirin on disability-free survival. ${ }^{15-17}$ The ASPREE study design, ${ }^{18} 19$ recruitment ${ }^{20}$ and baseline characteristics ${ }^{14}$ have been published previously. Participants had no previous diagnosis of atherosclerotic or atherothrombotic cardiovascular disease events, including myocardial infarction; heart failure; angina; pectoris; stroke or transient ischaemic attack or diagnosis of atrial fibrillation or high blood pressure..$^{21}$ Diagnosis of dementia or other serious illness likely to cause death within 5 years were also exclusion criteria. Genetic analysis was conducted on 13131 samples provided by Australian ASPREE participants aged 70 years or older at enrolment. ${ }^{22}$

\section{DNA sequencing and variant analysis}

A targeted sequencing panel was designed containing the $P C S K 9$ and $A P O B$ genes. ${ }^{22}$ Following standard protocols, DNA was extracted and sequenced using the Thermo Fisher Scientific S5TM XL system to average 200× depth, with sequences aligned to the human genome reference 37 . We identified candidate cholesterol-lowering variants from sequence data using two methods: (1) prediction of rare candidate LoF, protein-truncating $P C S K 9$ and $A P O B$ variants using the Loss-of-Function Transcript Effect Estimator tool, high-confidence filter, a plugin of Ensemble Variant Effect Predictor ${ }^{23}$ and (2) assessment of candidate variants associated with hypercholesterolaemia or hypobetalipoproteinaemia identified from published functional and population studies ${ }^{3024}$ (for list of variants meeting the inclusion criteria, see online supplemental materials). We also examined the effect of the more common PCSK9 R46L variant with known lipid-lowering effect $(\mathrm{rs} 11591147-\mathrm{T}) .^{25}$ Rare variants were curated manually by two or more laboratory scientists following the American College of Medical Genetics/Association for Molecular Pathology Standards ${ }^{26}$ and variant classification was agnostic to lipid effects. A random selection of $10 \%$ of rare variants detected were validated using Sanger sequencing with $100 \%$ concordance. The minor allele frequency (MAF) of each variant detected in the ASPREE population was compared with the MAF reported in the genome Aggregation Database Non-Finnish European reference population (gnomAD-NFE) ${ }^{23}$

\section{Association of variant carrier status with blood lipid levels}

We sought to determine whether $P C S K 9 / A P O B$ variant carriers in the ASPREE cohort were associated with lower serum cholesterol levels at the time of enrolment, versus age-matched and gender-matched non-carriers. To do this, we compared serum LDL-C and TC levels between detected variant carriers versus $n=9540$ age-matched and gendermatched non-carrier ASPREE controls who did not carry any $P C S K 9$ or $A P O B$ variants that met our inclusion criteria. Baseline LDL-C and TC levels were measured in routine blood samples provided by ASPREE participants at enrolment, analysed at commercial pathology laboratories. We tested association of variant carrier status with serum LDL-C and TC levels using multivariable linear regression, adjusting for age, gender, diabetes, hypertension, smoking status, alcohol use and body mass index (BMI). We first tested association using raw unadjusted LDL-C and TC levels (not accounting for statin use), then separately using statin-adjusted levels, dividing LDL-C and TC levels by 0.7 and 0.8 , respectively for

Table 1 Characteristics of sequenced participants at enrolment

ASPREE $\mathrm{N}=13131$ (mean age 75 years)

\begin{tabular}{|ll|}
\hline Female sex-no. (\%) & $7056(54)$ \\
\hline Age in years-no. $(\%)$ & \\
\hline $70-74$ & $7894(60)$ \\
$75-79$ & $3406(26)$ \\
$\geq 80$ & $1831(14)$ \\
\hline
\end{tabular}

Race or ethnic group*-no. (\%)

\begin{tabular}{lc}
\multicolumn{1}{l}{ White/Caucasian } & $12953(99)$ \\
\hline Other & $178(1)$ \\
Obese $\left(\mathrm{BMI} \geq 30 \mathrm{~kg} / \mathrm{m}^{2}\right) \dagger(\%)$ & 28 \\
Current smoking $(\%)$ & 4 \\
Statin use $(\%)$ & 33 \\
Heart, stroke or vascular disease§ (\%) & 0 \\
$\begin{array}{l}\text { Serum low-density lipoprotein } \\
\text { cholesterol—mean, mg/dL }\end{array}$ & 119 \\
Serum total cholesterol—mean, mg/dL & 204 \\
\hline
\end{tabular}

Sequenced individuals were Australian participants enrolled in the ASPREE clinical trial, aged 70 years and older (average age 75 years), without a previous diagnosis of cardiovascular disease events, dementia, permanent physical disability or current diagnosis of life-threatening cancer. Most were white/Caucasian and $54 \%$ were female.

*Self-report.

†Obese was defined as body mass index (weight $(\mathrm{kg}) /$ height $\left(\mathrm{m}^{2}\right)$ ) of $\geq 30$.

$\ddagger$ Anatomical Therapeutic Chemical code C10 (lipid-modifying agents).

§Baseline characteristics of participants in the ASPREE study. ${ }^{14}$ ASPREE, ASPirin in Reducing Events in the Elderly. 


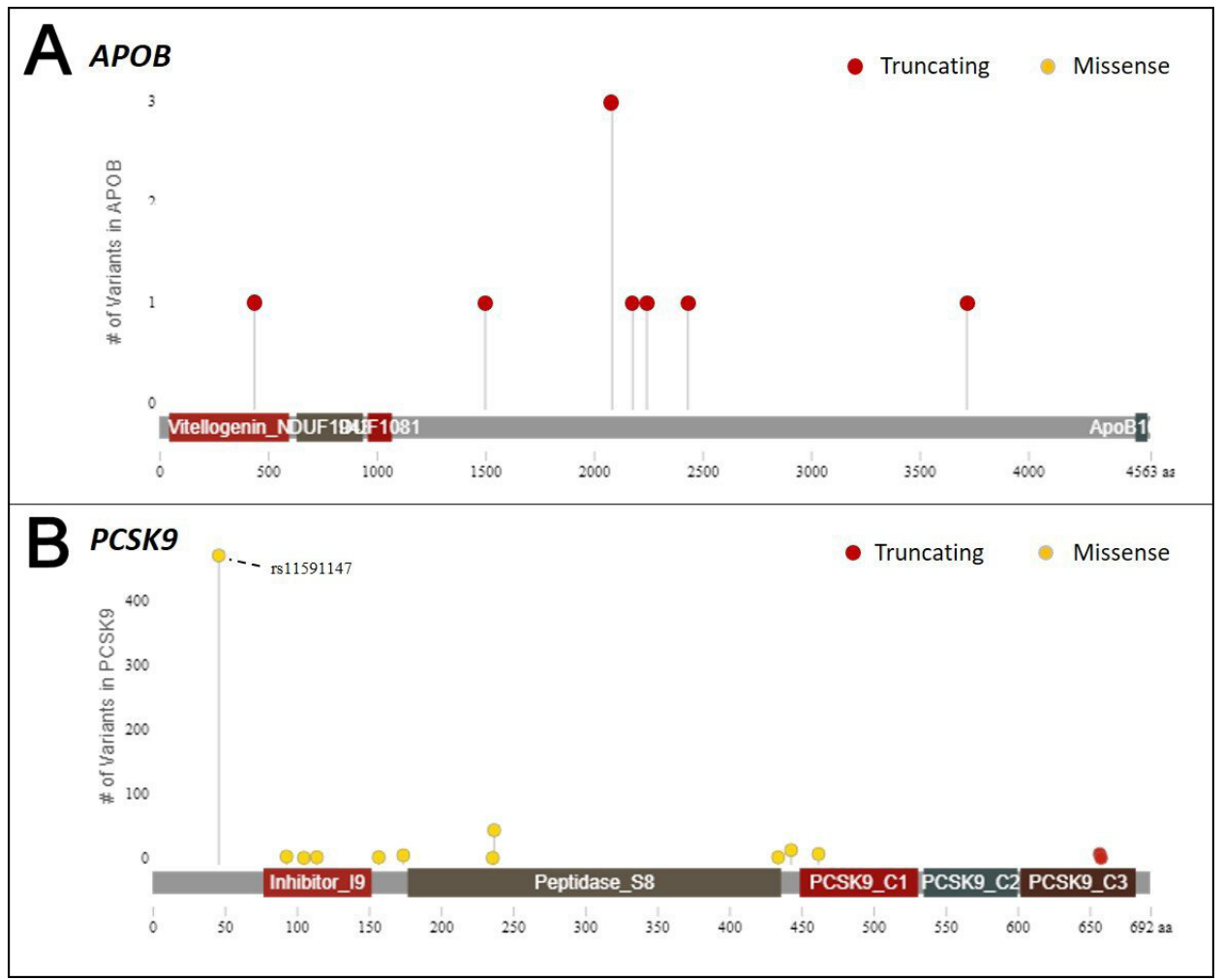

Figure 1 Schematic representation of the apolipoprotein $\mathrm{B}(A P O B)$ and proprotein convertase subtilisin kexin type 9 (PCSK9) genes indicating the location of identified variants. We detected 22 different rare protein truncating or missense $A P O B$ and PCSK9 variants with putative loss-of-function and lipid-lowering effect. We also investigated the more common PCSK9 R46L variant (rs11591147). Variants were found across different protein domains of the APOB and PCSK9 genes.

those using statin medication to estimate untreated levels, as done previously. ${ }^{3}$ We identified statin users based on concomitant medication data collected by ASPREE (Anatomical Therapeutic Chemical (ATC) code=C10, lipid-modifying agents).

\section{RESULTS}

Characteristics of the 13131 sequenced participants are shown in table 1 . The median age at enrolment was 75 years, with $54 \%$ of participants female; $28 \%$ obese and $4 \%$ current smokers. Participants had no previous diagnosis of cardiovascular disease or dementia. ${ }^{14}$ Most participants were of European ancestry (99\% self-reported as white/ Caucasian). The mean serum LDL-C and TC concentrations of the study population were 119 and $204 \mathrm{mg} / \mathrm{dL}$, respectively at the time of enrolment.

Among this population, we detected a total of 104 healthy older CHD-free individuals carrying rare candidate LoF variants that met our inclusion criteria (MAF $<0.01)$. Variants were found across multiple different domains of the $A P O B$ and $P C S K 9$ genes (schematic representation in figure 1). Overall, the carrier rate of detected rare variants in PCSK9/APOB meeting our inclusion criteria (when considered as a group) was 1 in 126 participants or $0.8 \%$.

We detected a total of 22 different rare $A P O B / P C S K 9$ variants, ranging in frequency between $\mathrm{MAF}=0.00335$ (detected in 44 ASPREE participants) and MAF $=0.00008$ (singletons detected in only one ASPREE participant) (table 2). MAFs of the variants detected in the ASPREE population were found to be consistently higher when compared with MAFs from the gnomAD-NFE reference population (table 2). We found six putatively novel rare $A P O B$ variants that, at the time of our analysis, were not found in the gnomAD or dbSNP databases, and were not previously reported in the literature. Each of these rare $A P O B$ variants were detected as singletons in the ASPREE cohort $(\mathrm{MAF}=0.0008)$ and met our criteria for $\mathrm{LoF}$ (table 2).

The serum LDL-C and TC concentrations for carriers of rare $A P O B$ or $P C S K 9$ variants were found to be consistently lower than the $n=9450$ non-carrier controls in the study (figure 2, table 2). For some rare variants, the reduction in LDL-C was up to $77.4 \mathrm{mg} / \mathrm{dL}$ (eg, $A P O B$ c.7300C $>\mathrm{T}$ (p.Gln2434Ter)). Based on raw LDL-C and TC levels uncorrected for statin use, rare variant heterozygous carrier status in ASPREE (when considered as a group of $n=104$ participants) was associated with $11.6 \mathrm{mg} / \mathrm{dL}(9.7 \%)$ lower serum LDL-C $(\mathrm{p}<0.001)$ and $7.8 \mathrm{mg} / \mathrm{dL}(3.9 \%)$ lower serum TC $(\mathrm{p}<0.001)$ versus noncarriers, adjusted for age, gender, diabetes, hypertension, smoking status, alcohol use and BMI (figure 3, table 3).

After adjusting for statin use (dividing LDL-C and TC levels by 0.7 and 0.8 , respectively for those taking statin medication), rare variant heterozygous carrier status was associated with $19.4 \mathrm{mg} / \mathrm{dL}(14.6 \%)$ lower adjusted serum LDL-C $(\mathrm{p}<0.001)$ and $16.4 \mathrm{mg} / \mathrm{dL}(7.5 \%)$ lower adjusted serum 


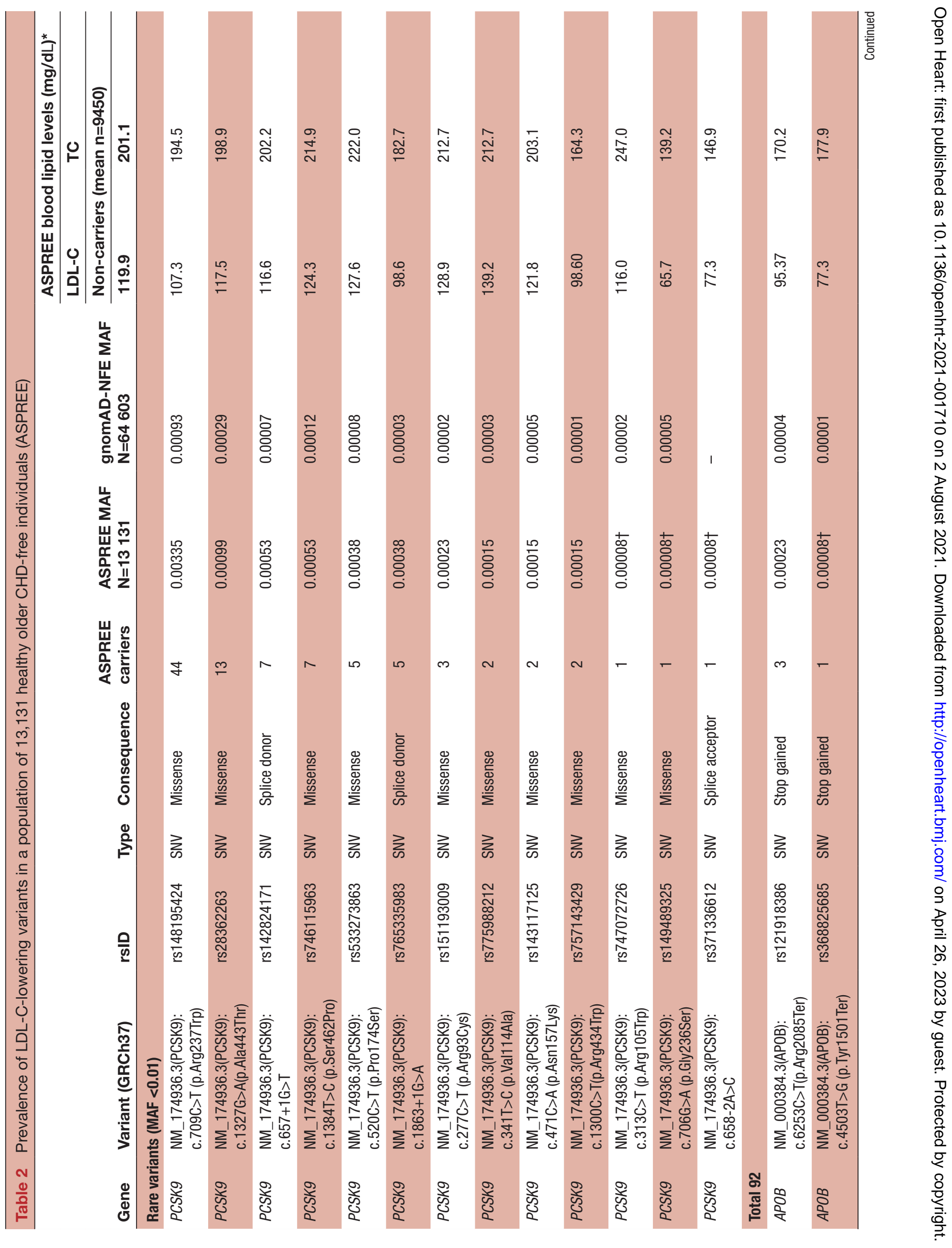




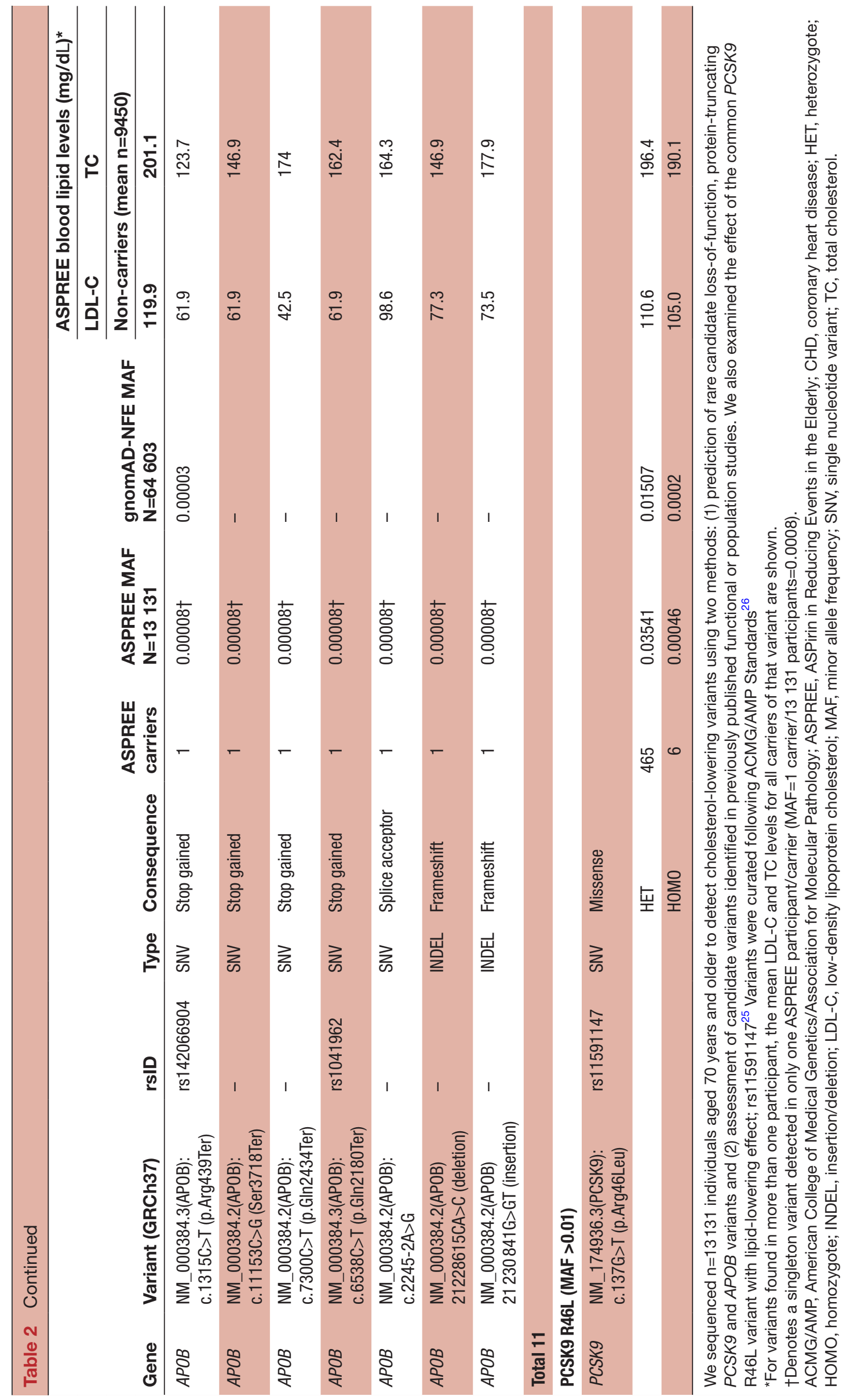




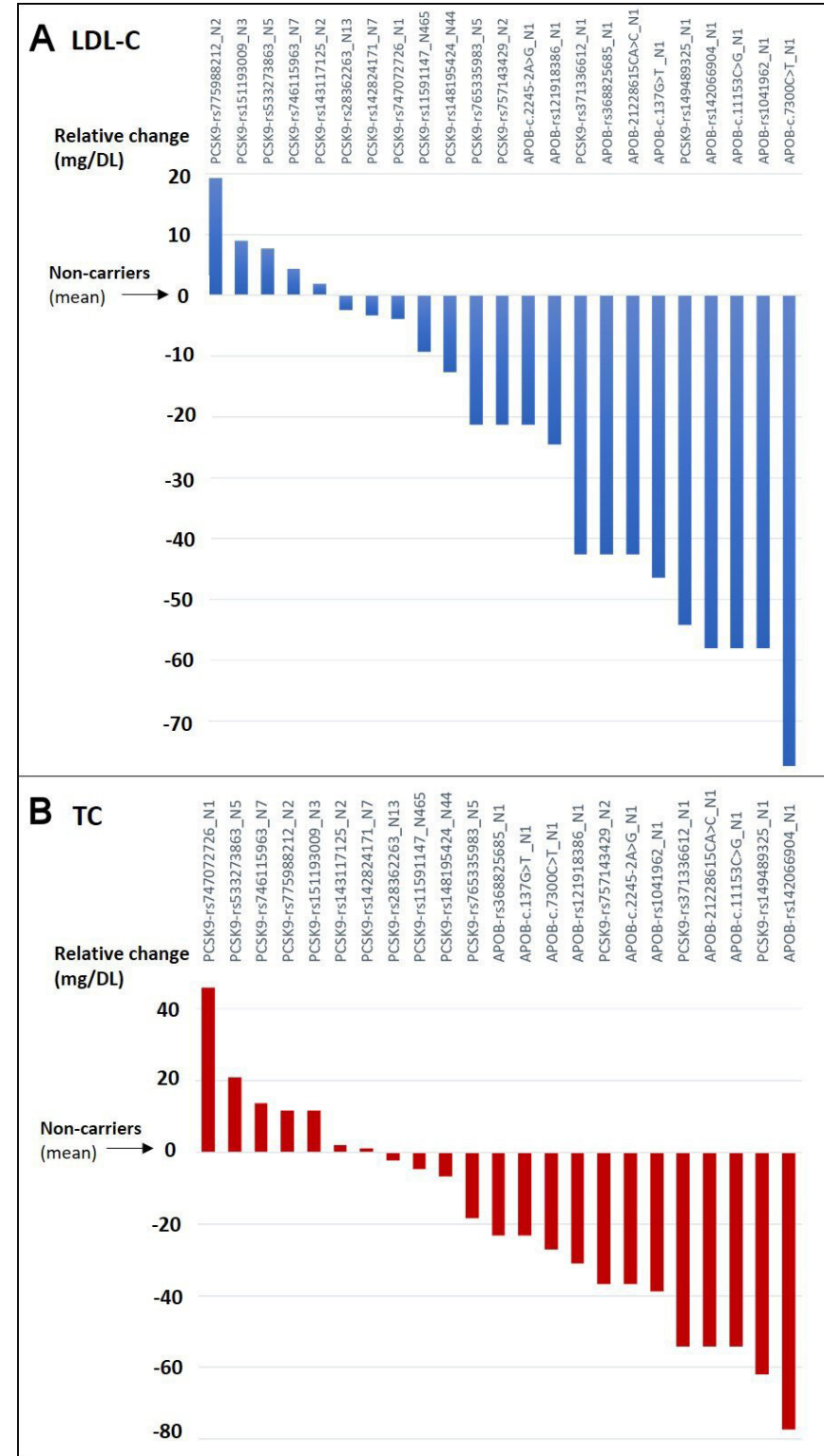

Figure 2 Relative changes in serum low-density lipoprotein cholesterol (LDL-C) and total cholesterol (TC) levels per variant detected, in variant carriers versus non-carriers. Shown are waterfall plots of the relative changes in serum LDL-C (A) and TC (B) concentrations at enrolment (mg/ $\mathrm{dL}$ ) for carriers of proprotein convertase subtilisin kexin type 9 (PCSK9) and apolipoprotein B (APOB) variants, compared with the mean concentration from $n=9450$ noncarrier controls (normalised to $0 \mathrm{mg} / \mathrm{dL}$ ). Variant name labels are shown vertically above the plots, and include the total number $(\mathrm{N})$ of carriers detected per variant. For variants detected in more than one participant, the mean LDL-C and TC levels for all carriers of that variant are plotted.

TC $(\mathrm{p}<0.001)$ versus non-carriers. The prevalence of lipidlowering statin prescriptions among rare variant carriers was $16 \%(\mathrm{n}=17 / 104)$, which was lower than that observed in noncarriers $(35 \%, \mathrm{n}=3324 / 9540)$.

The estimated median untreated LDL-C level in noncarriers was $131.5 \mathrm{mg} / \mathrm{dL}$ (110.4-154.7), compared with $112.1 \mathrm{mg} / \mathrm{dL}(92.8-139.2)$ in rare $P C S K 9 / A P O B$ variant
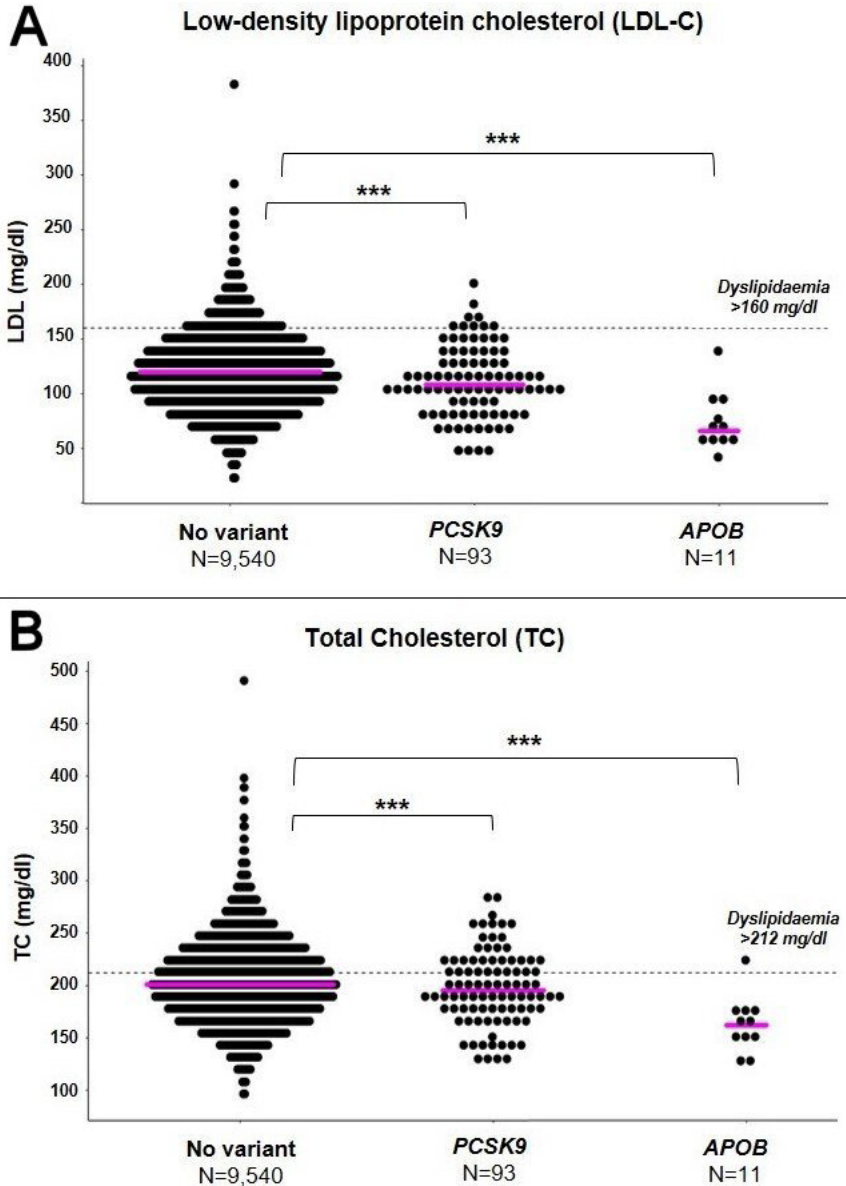

Figure 3 Proprotein convertase subtilisin kexin type 9 (PCSK9) and apolipoprotein $\mathrm{B}(A P O B)$ rare variant carrier status in ASPirin in Reducing Events in the Elderly (ASPREE) is associated with reduced serum low-density lipoprotein cholesterol (LDL-C) and total cholesterol (TC) levels. Shown is a comparison of serum LDL-C (A) and TC (B) levels at enrolment for carriers of rare variants (minor allele frequency (MAF) <0.01) in PCSK9 $(\mathrm{n}=93)$ and APOB $(\mathrm{n}=11)$, compared with non-carrier controls $(n=9540)$ from the ASPREE study. Results indicate that rare $A P O B / P C S K 9$ rare variant carrier status is associated with reduced serum LDL-C and TC levels versus non-carriers. Linear regression was used to compare statistical differences between groups adjusting for covariates. Median values are shown as coloured solid lines (magenta). Statistical significance is denoted as ${ }^{* \star *} \mathrm{p} \leq 0.001$. Dyslipidaemia (dotted lines) is defined as serum LDL-C $>160 \mathrm{mg} / \mathrm{dL}$, or serum TC $>212 \mathrm{mg} / \mathrm{dL} .{ }^{14}$ To convert values from $\mathrm{mg} / \mathrm{dL}$ to $\mathrm{mmol} / \mathrm{L}$ multiply by 0.02586 .

carriers. At the per-gene level, rare variant carrier status for $P C S K 9$ and $A P O B$ variants separately was also associated with significantly lower LDL-C levels for both genes $(\mathrm{p}<0.001)$.

For the more common PCSK9 R46L variant (rs11591147-T, ASPREE MAF=0.03541), heterozygous and homozygous carrier status were associated with $15.5 \mathrm{mg} / \mathrm{dL}(11.8 \%)$ lower and $25.2 \mathrm{mg} / \mathrm{dL}(19.2 \%)$ lower statin-corrected LDL-C levels, respectively $(\mathrm{p} \leq 0.001)$ (table 3). 
Table 3 Variant carrier status is associated with reduced serum LDL-C and TC levels

\begin{tabular}{|c|c|c|c|c|}
\hline $\begin{array}{l}\text { Rare variant group } \\
\text { PCSK } 9 / A P O B \\
\text { MAF }<0.01\end{array}$ & $\begin{array}{l}\text { Non-carriers } \\
\mathrm{N}=9540\end{array}$ & $\begin{array}{l}\text { Heterozygotes } \\
\mathrm{N}=104\end{array}$ & $\begin{array}{l}\text { Homozygotes } \\
\mathrm{N}=0\end{array}$ & $P$ value \\
\hline LDL-C (mg/dL) & $\begin{array}{l}119.9 \\
(96.7-143.1)\end{array}$ & $\begin{array}{l}108.3 \\
(81.2-131.5)\end{array}$ & - & $<0.001$ \\
\hline $\operatorname{adjLDL-C~(mg/dL)~}$ & $\begin{array}{l}131.5 \\
(112.1-150.8)\end{array}$ & $\begin{array}{l}112.1 \\
(92.8-139.2)\end{array}$ & - & $<0.001$ \\
\hline $\mathrm{TC}(\mathrm{mg} / \mathrm{dL})$ & $\begin{array}{l}201.1 \\
(177.9-228.2)\end{array}$ & $\begin{array}{l}193.3 \\
(170.1-220.4)\end{array}$ & - & $<0.001$ \\
\hline $\operatorname{adjTC~(mg/dL)~}$ & $\begin{array}{l}217.5 \\
(193.4-241.6)\end{array}$ & $\begin{array}{l}201.1 \\
(177.9-225.7)\end{array}$ & - & $<0.001$ \\
\hline $\begin{array}{l}\text { Common variant } \\
\text { PCSK9 rs11591147-T }\end{array}$ & $\begin{array}{l}\text { Non-carriers } \\
\mathrm{N}=9540\end{array}$ & $\begin{array}{l}\text { Heterozygotes } \\
\mathrm{N}=465\end{array}$ & $\begin{array}{l}\text { Homozygotes } \\
\mathrm{N}=6\end{array}$ & $P$ value \\
\hline LDL-C (mg/dL) & $\begin{array}{l}119.9 \\
(96.7-143.1)\end{array}$ & $\begin{array}{l}112.1 \\
(88.9-131.5)\end{array}$ & $\begin{array}{l}106.35 \\
(95.7-117.0)\end{array}$ & $<0.001$ \\
\hline $\operatorname{adjLDL-C~(mg/dL)~}$ & $\begin{array}{l}131.5 \\
(112.1-150.8)\end{array}$ & $\begin{array}{l}116.0 \\
(96.7-135.3)\end{array}$ & $\begin{array}{l}106.4 \\
(95.7-117.0)\end{array}$ & $<0.001$ \\
\hline $\mathrm{TC}(\mathrm{mg} / \mathrm{dL})$ & $\begin{array}{l}201.1 \\
(177.9-228.2)\end{array}$ & $\begin{array}{l}197.2 \\
(170.1-216.6)\end{array}$ & $\begin{array}{l}197.2 \\
(168.2-211.7)\end{array}$ & $<0.001$ \\
\hline $\operatorname{adjTC~(mg/dL)~}$ & $\begin{array}{l}217.5 \\
(193.4-241.6)\end{array}$ & $\begin{array}{l}201.1 \\
(183.6-227.1)\end{array}$ & $\begin{array}{l}197.2 \\
(168.2-211.7)\end{array}$ & $<0.001$ \\
\hline
\end{tabular}

We compared serum LDL-C and TC levels between variant carriers and $n=9540$ non-carrier controls from the ASPREE study. The multivariable linear regression model adjusted for age, gender, diabetes, hypertension, smoking status, alcohol use and body mass index. We first tested association using raw unadjusted LDL-C and TC levels (not accounting for statin use), then separately using statin-adjusted levels (adjLDL-C, adjTC), dividing LDL-C and TC levels by 0.7 and 0.8 , respectively for those using statin medication to estimate untreated levels, as done previously. ${ }^{3}$ We identified statin users based on concomitant medications recorded by ASPREE at baseline. Data are presented as median (IQR). Statistical significance is denoted by $p<0.001$. To convert values from $\mathrm{mg} / \mathrm{dL}$ to mmol/L multiply by 0.02586 . ATC code C10 (lipid-modifying agents).

ASPREE, ASPirin in Reducing Events in the Elderly; ATC, Anatomical Therapeutic Chemical; LDL-C, low-density lipoprotein cholesterol; MAF, minor allele frequency; TC, total cholesterol.

\section{DISCUSSION}

In this study, we identified rare $P C S K 9$ and $A P O B$ variants that are associated with lower serum LDL-C levels among a population of healthy older individuals without a history of CHD events. Most of the variants detected were enriched in frequency among the healthy older CHD-free ASPREE population, compared with a large reference population of similar genetic ancestry (gnomAD-NFE). Serum LDL-C and TC concentrations for rare variant carriers were consistently lower than non-carriers in the study, and rare variant carrier status was associated with $19.4 \mathrm{mg} / \mathrm{dL}(14.6 \%)$ lower serum LDL-C and TC concentrations, after adjusting for statin use. The prevalence of statin prescriptions in variant carriers were less than half that observed in non-carriers. Together, these results indicate that lipid-lowering genetic variants are enriched in healthy older individuals without CHD, and play a role in coronary disease-free survival throughout the human lifespan.

Consistent with other studies, ${ }^{1-9}$ we observed that LoF variants in the PCSK9 and $A P O B$ gene are associated with lower serum LDL-C concentrations. However, to our knowledge, the prevalence shown in ASPREE during ageing free of atherosclerotic cardiovascular disease manifestations has not previously been demonstrated. Variants analysed were found to lower serum LDL-C and TC levels among the ASPREE population, with the difference in LDL-C concentrations between ASPREE rare variant carrier and noncarriers being $\sim 20 \mathrm{mg} / \mathrm{dL}$ after adjusting for statin use. At an average participant age of 75 years, this represents the effect of potentially a lifetime of exposure to genetically determined lower LDL-C.

Meta-analysis of statin trials suggests for a $38 \mathrm{mg} /$ $\mathrm{dL}$ reduction in $\mathrm{LDL}-\mathrm{C}$, there is a $20 \%-22 \%$ reduction in CHD risk, in the setting of relatively short clinical trials. ${ }^{27}$ Mendelian randomisation studies, however, demonstrate the importance of lifetime exposure to low LDL-C, suggesting that genetically determined low LDL-C is associated with a greater magnitude of CHD risk-reduction, compared with equivalent reduction through statin use. ${ }^{28}$ It is therefore likely that ASPREE rare variant carriers detected in this study, who have experienced a lifetime of exposure to genetically lower LDL-C, have benefited substantially from lower CHD risk. However, it is noteworthy that the rare variants detected likely account for only a fraction of the reduced CHD risk in the ASPREE population $(n=13131)$, with a range of other genetic and lifestyle factors contributing. 
Strengths of the study include the sample size and unique ascertainment of the ASPREE population. The sequenced cohort comprised 13131 individuals with an average age of 75 years, with no previous diagnosis of CHD or other cardiovascular events. It is rare for a population ascertained with these characteristics to be made available for genetic analysis. The sequenced cohort was the result of a unique set of circumstances made possible by the strict ASPREE inclusion criteria and age cut-off, and associated research biobank. ${ }^{1429}$

Another strength of the study is that ASPREE participants were well characterised, each receiving a medical assessment by a general practitioner at enrolment, to confirm eligibility for the trial, and to rule out previous diagnoses of CHD. ${ }^{14}$ This provided confidence that detected variant carriers were CHD event-free at enrolment. Other strengths of the study include the depth of sequencing, focus on canonical lipid metabolism genes with established biological effect and stringency of variant curation used, to ensure only high-confidence variants were included in analyses.

Limitations of the study include our results not necessarily being generalisable to populations of non-European ancestry. Furthermore, we caution the comparison of rare variant prevalence between ASPREE and reference populations such as gnomAD, due to the potential for technical artefacts introduced by differences in sequencing technologies and variant curation, and population stratification related to differences in genetic ancestry. These well-known sources of variability are compounded when attempting to compare rare variant frequencies between studies. ${ }^{30}$ Nonetheless, our results suggest that rare PSCK9/ $A P O B$ variants are enriched in healthy older CHD-free individuals (table 2), consistent with previous studies showing that LDL-C-lowering variants are associated with reduced risk for CHD and longevity. ${ }^{1-9}$

Regarding the genes analysed in our study, we focused on only the most established two canonical lipid metabolism genes where rare LoF variants have been demonstrated to have a high effect in reducing LDL-C levels (PCSK9 and APOB). ${ }^{1389}$ We did not examine gain-offunction variants in the $L D L R$ gene, or LoF variants in other genes that have been associated with LDL-C reduction (eg, NPC1L1, LPA, APOC3, ANGPTL3/4 and ASGR1) as these genes were not included on the sequencing panel used. We also could not calculate polygenic scores due to the targeted nature of the sequencing assay, and could not assess subclinical atherosclerosis due to absence of data on coronary artery calcium and carotid intima-media thickness. We did not functionally validate the detected rare variants.

Our study demonstrates the unique contribution of healthy older populations to exploring genetic determinants of health and lifespan. Historically, healthy older populations have notbeen the focus oflarge humangenetic studies, mainly due to the difficulties in ascertaining large numbers of samples in this age group. However, studies focused on well-characterised populations of healthy older individuals represent an underexplored opportunity for the detection and improved understanding of protective genetic variants, especially in the context of lipid regulation and $\mathrm{CHD} .{ }^{29}$ An improved understanding of the role played by protective lipid-lowering variants may help inform novel future approaches to CHD riskreduction and lipid management.

\section{Author affiliations}

${ }^{1}$ Department of Epidemiology and Preventive Medicine, School of Public Health and Preventive Medicine, Monash University, Melbourne, Victoria, Australia

${ }^{2}$ Department of Genetics and Genomic Sciences, Icahn School of Medicine at Mount Sinai, New York, New York, USA

${ }^{3}$ School of Medicine, Faculty of Medicine and Health Sciences, The University of Western Australia, Perth, Western Australia, Australia

${ }^{4}$ Department of Clinical Biochemistry, PathWest Laboratory Medicine WA, Royal Perth Hospital and Fiona Stanley Hospital Network, Royal Perth Hospital, Perth, Western Australia, Australia

${ }^{5}$ School of Public Health, Curtin University, Perth, Western Australia, Australia ${ }^{6}$ Berman Center for Outcomes and Clinical Research, Hennepin Healthcare Research Institute, Hennepin Healthcare, Minneapolis, Minnesota, USA

${ }^{7}$ Monash Cardiovascular Research Centre, Monash University and MonashHeart, Monash Health, Clayton, Victoria, Australia

${ }^{8}$ Lipid Disorders Clinic, Department of Cardiology, Royal Perth Hospital, Perth, Western Australia, Australia

Acknowledgements We thank the ASPREE trial staff in Australia and the USA, the ASPREE participants who volunteered for the trial and the general practitioners and staff of the medical clinics who cared for the participants.

Contributors PL, MR, RS, AJH, JP, GP, ES and JJMcN made substantial contributions to conception and design, acquisition of data or analysis and interpretation of data. PL, MR, AJH, JT, AT, CR, SZ, AMM, SN, GW and JJMcN were involved in drafting the manuscript or revising it critically for important intellectual content. All authors gave final approval of the version to be published. $\mathrm{PL}$ and JJMCN agreed to be accountable for all aspects of the work in ensuring that questions related to the accuracy or integrity of any part of the work are appropriately investigated and resolved.

Funding The ASPREE Healthy Ageing Biobank is supported by a Flagship cluster grant (including the Commonwealth Scientific and Industrial Research Organisation, Monash University, Menzies Research Institute, Australian National University, University of Melbourne); and grants (U01AG029824) from the National Institute on Aging and the National Cancer Institute at the National Institutes of Health, by grants (334047 and 1127060) from the National Health and Medical Research Council of Australia, and by Monash University and the Victorian Cancer Agency. PL is supported by a National Heart Foundation Future Leader Fellowship (102604).

Competing interests GW has received honoraria and/or research grants from Arrowhead, AstraZeneca, Kowa, Regeneron, Sanofi, Amgen and Novartis. SN has received research support and/or honoraria for Amgen, AstraZeneca, Eli Lilly, Esperion, Novartis, Merck, Pfizer, lowa and Sanofi-Regeneron. RS serves as VicePresident of Technology Development at Sema4. ES serves as Chief Executive Officer at Sema4. No other conflicts were reported.

Patient consent for publication Not required.

Ethics approval The study received approvals from the Alfred Hospital Research Ethics Committee (Project 390/15). All participants provided written informed consent for genetic research.

Provenance and peer review Not commissioned; externally peer reviewed.

Data availability statement Data are available from the corresponding author on request.

Open access This is an open access article distributed in accordance with the Creative Commons Attribution Non Commercial (CC BY-NC 4.0) license, which permits others to distribute, remix, adapt, build upon this work non-commercially, and license their derivative works on different terms, provided the original work is 
properly cited, appropriate credit is given, any changes made indicated, and the use is non-commercial. See: http://creativecommons.org/licenses/by-nc/4.0/.

ORCID iDs

Paul Lacaze http://orcid.org/0000-0002-0902-6798

Jing Pang http://orcid.org/0000-0002-9700-6948

\section{REFERENCES}

1 Cohen JC, Boerwinkle E, Mosley TH, et al. Sequence variations in PCSK9, low LDL, and protection against coronary heart disease. $N$ Engl J Med 2006;354:1264-72.

2 Dewey FE, Gusarova V, O'Dushlaine C, et al. Inactivating variants in ANGPTL4 and risk of coronary artery disease. N Engl J Med 2016;374:1123-33.

3 Peloso GM, Nomura A, Khera AV, et al. Rare protein-truncating variants in $\mathrm{ApoB}$, lower low-density lipoprotein cholesterol, and protection against coronary heart disease. Circ Genom Precis Med 2019;12:e002376.

4 Ference BA, Robinson JG, Brook RD, et al. Variation in PCSK9 and HMGCR and risk of cardiovascular disease and diabetes. $N$ Engl J Med 2016;375:2144-53.

5 Nomura A, Won $\mathrm{H}-\mathrm{H}$, Khera AV, et al. Protein-truncating variants at the cholesteryl ester transfer protein gene and risk for coronary heart disease. Circ Res 2017;121:81-8.

6 Ference BA, Kastelein JJP, Ray KK, et al. Association of triglyceridelowering LPL variants and LDL-C-lowering LDLR variants with risk of coronary heart disease. JAMA 2019;321:364-73.

7 Ye H, Zhao Q, Huang Y, et al. Meta-analysis of low density lipoprotein receptor (LDLR) rs2228671 polymorphism and coronary heart disease. Biomed Res Int 2014;2014:564940

8 Burnett JR, Hooper AJ. Common and rare gene variants affecting plasma LDL cholesterol. Clin Biochem Rev 2008;29:11-26.

9 Rimbert A, Pichelin M, Lecointe S, et al. Identification of novel ApoB mutations by targeted next-generation sequencing for the molecular diagnosis of familial hypobetalipoproteinemia. Atherosclerosis 2016;250:52-6.

10 Dron JS, Hegele RA. Complexity of mechanisms among human proprotein convertase subtilisin-kexin type 9 variants. Curr Opin Lipidol 2017;28:161-9.

11 Barzilai N, Atzmon G, Schechter C, et al. Unique lipoprotein phenotype and genotype associated with exceptional longevity. JAMA 2003;290:2030-40.

12 Welty FK. Hypobetalipoproteinemia and abetalipoproteinemia. Curr Opin Lipidol 2014;25:161-8.

13 Graham I, Shear C, De Graeff P, et al. New strategies for the development of lipid-lowering therapies to reduce cardiovascular risk. Eur Heart J Cardiovasc Pharmacother 2018;4:119-27.

14 McNeil JJ, Woods RL, Nelson MR, et al. Baseline characteristics of participants in the ASPREE (aspirin in reducing events in the elderly) study. J Gerontol A Biol Sci Med Sci 2017;72:1586-93.
15 McNeil JJ, Wolfe R, Woods RL, et al. Effect of aspirin on cardiovascular events and bleeding in the healthy elderly. $N$ Engl J Med 2018;379:1509-18.

16 McNeil JJ, Woods RL, Nelson MR, et al. Effect of aspirin on disability-free survival in the healthy elderly. $N$ Engl J Med 2018;379:1499-508

17 McNeil JJ, Nelson MR, Woods RL, et al. Effect of aspirin on all-cause mortality in the healthy elderly. N Engl J Med 2018;379:1519-28.

18 ASPREE Investigator Group. Study design of aspirin in reducing events in the elderly (ASPREE): a randomized, controlled trial. Contemp Clin Trials 2013;36:555-64.

19 Nelson MR, Reid CM, Ames DA, et al. Feasibility of conducting a primary prevention trial of low-dose aspirin for major adverse cardiovascular events in older people in Australia: results from the aspirin in reducing events in the elderly (ASPREE) pilot study. Med $\mathrm{J}$ Aust 2008;189:105-9.

20 Lockery JE, Collyer TA, Abhayaratna WP, et al. Recruiting general practice patients for large clinical trials: lessons from the aspirin in reducing events in the elderly (ASPREE) study. Med $\mathrm{J}$ Aust 2019;210:168-73.

21 Lacaze P, Sebra R, Riaz M, et al. Familial hypercholesterolemia in a healthy elderly population. Circ Genom Precis Med 2020;13:e002938.

22 Lacaze P, Sebra R, Riaz M, et al. Medically actionable pathogenic variants in a population of 13,131 healthy elderly individuals. Genet Med 2020;22:1883-6.

23 Lek M, Karczewski KJ, Minikel EV, et al. Analysis of protein-coding genetic variation in 60,706 humans. Nature 2016;536:285-91.

24 Elbitar S, Susan-Resiga D, Ghaleb Y, et al. New sequencing technologies help revealing unexpected mutations in autosomal dominant hypercholesterolemia. Sci Rep 2018;8:1943.

25 Benn M, Nordestgaard BG, Grande P, et al. Pcsk9 R46L, low-density lipoprotein cholesterol levels, and risk of ischemic heart disease: 3 independent studies and meta-analyses. J Am Coll Cardiol 2010;55:2833-42.

26 Richards S, Aziz N, Bale S, et al. Standards and guidelines for the interpretation of sequence variants: a joint consensus recommendation of the American College of Medical Genetics and Genomics and the Association for Molecular Pathology. Genet Med 2015;17:405-23.

27 Cholesterol Treatment Trialists' (CTT) Collaborators, Mihaylova B, Emberson J, et al. The effects of lowering LDL cholesterol with statin therapy in people at low risk of vascular disease: meta-analysis of individual data from 27 randomised trials. Lancet 2012;380:581-90

28 Ference BA, Yoo W, Alesh I, et al. Effect of long-term exposure to lower low-density lipoprotein cholesterol beginning early in life on the risk of coronary heart disease: a Mendelian randomization analysis. Am Coll Cardiol 2012;60:2631-9.

29 Lacaze P, Woods R, Zoungas S, et al. The genomic potential of the aspirin in reducing events in the elderly and statins in reducing events in the elderly studies. Intern Med J 2017;47:461-3.

30 Lee S, Abecasis GR, Boehnke M, et al. Rare-variant association analysis: study designs and statistical tests. Am J Hum Genet 2014;95:5-23. 